\title{
SCM instance computer disruptions concept Lecture Application
}

\author{
Kunliang $\mathrm{XU}^{1,}$, , Yanlin $\mathrm{TAO}^{1, \mathrm{~b}}$ \\ ${ }^{1}$ School of Computer Science and Engineering, Qujing Normal University, Yunnan 655011, China; \\ a422078334@qq.com, \\ b401004120@ qq.com
}

Keywords: SCM Examples, Interrupt concept, Application.

\begin{abstract}
In this paper, the microcontroller interrupt control technology, designed a simple microcontroller to control the LED lights off simulation system, the system was applied to the lectures can be intuitive so that students understand the process of computer disruptions, achieve a multiplier effect of teaching.
\end{abstract}

\section{Introduction}

CPU During normal operating procedures, due to internal or external events cause the CPU to suspend execution of the current program execution and implementation instead deal with new situations. After the treatment, and then return to the original program execution. A call to an interrupt is an external event occurs when the corresponding processing program (or service) process. Interrupt service routine and interrupt the CPU is running is independent, it does not pass data to each other. The specific process shown in Figure 1 by the following[1].

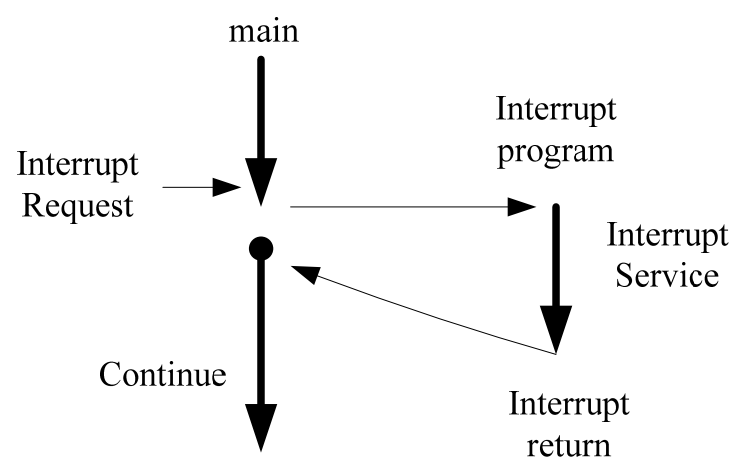

Fig.1 interrupt conceptual diagram

With the interrupt function, PC systems can make CPU and peripherals at the same time work in a timely manner so that the system can respond to external events. And with interrupt capability, CPU allows multiple peripherals simultaneously. This will greatly improve the utilization of CPU, but also improve the data input and output speed. On the other hand, with the interrupt function, it can make the CPU timely processing of all kinds of hardware and software failures. Computer during operation, often appear in advance or unforeseen circumstances some failures, such as power failure, memory error, arithmetic overflow and so on. Computer systems can use their own interrupt processing without stopping or reporting staff.Organization of the Text[2].

Therefore, the computer interrupts has an extremely important role in computer technology, but author with many years experience in teaching courses Principles of Computer Organization found that although cited to explain the concept of real-life examples to compare to understand, but students are still not fully understood, this article designed a simple microcontroller to control the LED lights off simulation system, the system was applied to the lectures can be intuitive so that students understand the process of computer disruptions, achieve a multiplier effect of teaching[2]. 


\section{System Design}

According to teaching requirements, the system should have the main analog functions and interrupt simulation. Design block diagram as shown in Figure 2.Page Numbers. Do not number your paper: All manuscripts must be in English, also the table and figure texts, otherwise we cannot publish your paper. Please keep a second copy of your manuscript in your office. When receiving the paper, we assume that the corresponding authors grant us the copyright to use the paper for the book or journal in question. Should authors use tables or figures from other Publications, they must ask the corresponding publishers to grant them the right to publish this material in their paper. Use italic for emphasizing a word or phrase[3].

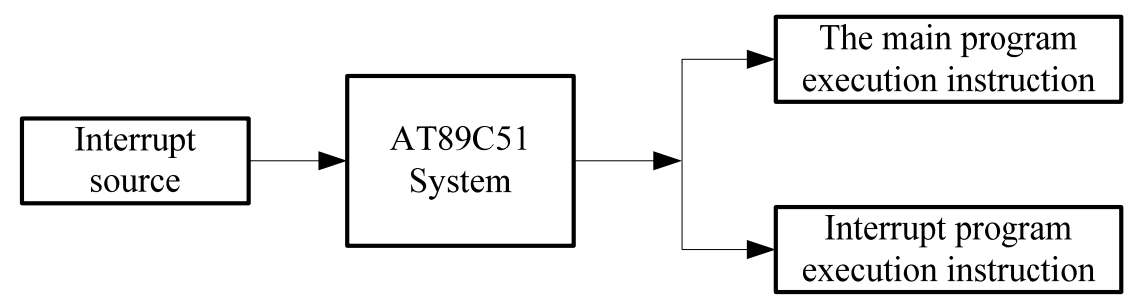

Fig.2 System Block Diagram Figure

\section{Introduce single chip}

As used herein, the chip is 80C51 series microcontroller, while AT89C51 microcontroller chip more popular in recent years. Atmel company developed and produced by the United States, the biggest feature is the inclusion of internal programming can be repeatedly flash memory Flash ROM, and can write a program easy to use. AT89C51 microcontroller pinout diagram shown in Figure 3 below[4].

\begin{tabular}{|c|c|c|c|}
\hline & & $\overline{P_{00}}$ & 39 \\
\hline & $P_{11}$ & $\mathrm{P}_{01}$ & $\frac{38}{37}$ \\
\hline & $\begin{array}{l}P_{12} \\
\mathbf{p}^{2}\end{array}$ & $\mathrm{P}_{02}$ & 36 \\
\hline & $\begin{array}{l}P_{13} \\
P_{14}\end{array}$ & $\mathrm{P}_{03}$ & 35 \\
\hline & $\mathbf{P}_{15}^{14}$ & $\mathbf{P}_{05}^{04}$ & 34 \\
\hline 8 & $P_{16}$ & $P_{06}$ & $\frac{33}{32}$ \\
\hline & & $P_{07}$ & \\
\hline 12 & $\mathrm{INT}_{1}$ & $\mathrm{P}_{20}$ & $\frac{21}{32}$ \\
\hline & $\mathrm{INT}_{0}$ & $\mathbf{P}_{21}$ & $\frac{22}{33}$ \\
\hline 15 & $\mathrm{~T}_{1}$ & $\begin{array}{l}P_{23}^{22} \\
P_{23}\end{array}$ & 24 \\
\hline 14 & $\mathrm{~T}_{0}$ & $\mathrm{P}_{24}$ & 25 \\
\hline 31 & $\overline{\mathrm{E}} \mathrm{A} / \mathrm{VP}$ & $\mathrm{P}_{25}$ & $\frac{26}{27}$ \\
\hline 19 & $X_{1}$ & $P_{27}$ & 28 \\
\hline 18 & $\mathrm{X}_{2}$ & & \\
\hline 9 & RESET & RXD & 10 \\
\hline 17 & $\overline{\mathrm{RD}}$ & TXD & $\frac{11}{30}$ \\
\hline 16 & $\overline{\mathrm{WR}}$ & PSEN & 29 \\
\hline
\end{tabular}

Fig.3 AT89C51 microcontroller pinout diagram

This article relates to the pin function as follows[4]:

VCC: power supply voltage.

GND: Ground.

P0 port: P0 port is an 8-bit open drain bidirectional I / O port, each pin can absorb 8TTL gate current. When the pin is $\mathrm{P} 0$ port of the first one to write one, it is defined as a high-impedance input. P0 can be used for external program data memory, which can be defined as the low eight bits of data 
/ address. When FIASH programming, P0 port as the original code input, when FIASH verify, P0 output of the original code, then $\mathrm{P} 0$ must be connected to an external pull-up resistor.

P3.2 / INT0 (External Interrupt 0)

RST: reset input. When the oscillator reset the device to maintain high time RST pin for two machine cycles.

/ EA / VPP: When / EA is held low, then during this period the external program memory $(0000 \mathrm{H}-\mathrm{FFFFH})$, regardless of whether there is an internal program memory. Note that encryption 1:00, / EA will be internally locked RESET; when

XTAL1: Input the inverting oscillator amplifier input and the internal clock operating circuit.

XTAL2: From inverted oscillator output.

\section{Hardware emulation circuit design}

This design uses Proteus 7.1 software receipt simulation schematic shown in Figure 4.



Fig. 4 Hardware simulation circuit

Hardware simulation circuit shown in Figure 4, wherein two LED lights (red and green LED1 LED2) connected to the microcontroller P0.0 and P0.1, after the program runs, connected to the green LED2 P0.1 lit in external interrupt P3.2 connect a button contact switch S1. When you press S1 simulate external interrupt to occur, then P3.3 = 0 , external interrupt is generated, so that the mouth is connected to the P0.0 LED1 is lit red, green LED2 goes off. After the delay 100MS, red LED1 light is off, the green LED2 lights. Representative processed interrupt service routine returns to the main program[5-6].

\section{Software design}

According to the functional requirements of the system, with hardware circuit connection diagram, in the Keil C51 programming and generate .HEX control program, then the program can be simulated load the microcontroller. Design procedures are as follows: 


\author{
ORG 0003H \\ AJMP LEDKZ \\ ORG 0100H \\ MAIN:SETB EA \\ SETB EX1 \\ SETB IT1 \\ LL:CLR P0.0 \\ SJMP LL \\ LEDKZ:SETB P0.0 \\ CLR P0.1 \\ LED1:MOV R4,\#200 \\ LED2:MOV R5,\#240 \\ DJNZ R5,\$ \\ DJNZ R4,LED2 \\ SETB P0.1 \\ RETI \\ END
}

\title{
Conclusion
}

In Proteus 7.1, click the Run button can be simulation results show that, first, green lights, when the switch S1 is pressed, an interrupt is generated, the green light off, red and other bright, analog interrupt service routine $100 \mathrm{MS}$, the red light is off, green light, can be a good simulation of the interrupt process is completed. Teaching the example application to interrupt concept computer composition principle, students can press the switch S1 in the simulation environment to appreciate and respond to the process generate an interrupt, so to explain the concept of a more intuitive, and achieved good teaching results.

\section{Acknowledgment}

This paper is supported by three project funds: Yunnan Provincial Department of Education Science Research Fund general project (project number: 2015Y432), 2015 Yunnan province college students innovation and entrepreneurship projects, Yunnan minority students in the mountains to the countryside, the electricity business venture research (project number: 115); the Ministry of education information technology Education Ministry of education, national education resources information direction (number: EIN2014001).

\section{References}

[1] Shuo Fei Tang,Computer Principle (2nd Edition), Higher Education Press,2008.p.194-201.

[2] Information on http://baike.baidu.com/subview/121718/5143456.htm

[3] NING Ai-min, "Single-chip Microcomputer Application Technology" Courses in Design of Multimedia Teaching Software and its Application, Higher Education Forum,(2012)No.9). p.115-117.

[4] Information on http://www.elecfans.com/book/story.php?id=372

[5] Jing Hua Li,Jun Cao,Based on analysis of 51 single-chip control system software delay program, Journal of Guilin University of Aerospace Technology,(2013)No.2,p.123-125

[6] Chun Lin Yang, Single chip microcomputer application technology project guide, Renmin University of China press, 2014 